\title{
Characterization of SiC/C Nanocomposite Powders Synthesized by Arc-Discharge
}

\author{
Lei Zhou, Jie Yi Yu, Jian Gao, Dong Xing Wang, Xiao Rong Gan, \\ Fang Hong Xue, Hao Huang, Xing Long Dong*
}

Key Laboratory of Materials Modification by Laser, Ion, and Electron Beams (Ministry of Education) and School of Materials Science and Engineering, Dalian University of Technology, Dalian 116024, China

*Correspondence to:

Dong XL,

Tel: +86-0411-84706130

Fax: +86-0411-84709284

E-mail: dongx|@dlut.edu.cn.

Received December 8, 2015

Revised December 9, 2015

Accepted December 9, 2015
In this paper, three carbon sources, i.e., solid graphite, gaseous $\mathrm{CH}_{4}$ and liquid ethanol, and one solid silicon source were employed to synthesize SiC/C nanocomposite powders by arc-discharge plasma. The processing conditions such as the component ratios of raw materials, atmospheric gases, etc. were adjusted for controllable synthesis of the nanopowders. It is indicated that both of solid graphite and silicon can be co-evaporated and reacted to form nanophases of cubic $\beta$-SiC with $\sim 50 \mathrm{~nm}$ in mean size and a little free graphite; the carbon atoms decomposed from gaseous $\mathrm{CH}_{4}$ favor to combine with the evaporated silicon atoms to form the dominant $\mathrm{SiC}$ nanophase; liquid carbon source of ethanol can also be used to harvest the main $\beta$-SiC and minor $6 \mathrm{H}$-SiC phases in the assembly of nanoparticles. The as-prepared $\mathrm{SiC} / \mathrm{C}$ nanocomposite powders were further purified by a heat-treatment in air and their photocatalytic performances were then greatly improved.

Key Words: Arc-discharge, Different carbon sources, SiC/C, Nanocomposite, Photocatalytic

\section{INTRODUCTION}

Since Fujishima and Honda found that $\mathrm{TiO}_{2}$ has photolysis of water in 1972 (Fujishima \& Honga, 1972), the potential applications of photocatalytic materials got more attention. A large numbers of semiconductor materials with wide band gap (Kang et al., 1999; Zhou et al., 2006) have be caused concern on their photocatalytic behaviors. As one of the third generation, the silicon carbides have been extensively investigated on the physical and chemical properties (Kang et al., 2007; Kang et al., 2009; Shao et al., 2009; Nacera et al., 2011; Liu et al., 2012), among them the photocatalytic properties attracted particular attention until recent years (Noboru et al., 2002; Nicolas et al., 2004; Isaias et al., 2013). Nariki et al. (1990) firstly reported the photocatalytic property of SiC synthesized by DC arc plasma method. They used the bulk $\mathrm{SiC}$ as raw material to produce $\mathrm{SiC}$ nanoparticles under the ambient atmosphere of hydrogen and inert gases with different ratios. It was found that the photocatalytic performance of $\mathrm{SiC}$ nanoparticles is comparably excellent as $\mathrm{Pt} / \mathrm{TiO}_{2}$ in the produced capacity of hydrogen, in which the productions were exposed to the ultraviolet irradiation in wavelength range of 210 to $460 \mathrm{~nm}$.

The microstructure of $\mathrm{SiC} / \mathrm{C}$ composite is beneficial in photocatalytic decomposition of the contaminants, due to the surface graphite layer with better conductivity can quickly deliver the generated photoelectrons away and significantly reduce the electron-hole complex, thus the efficiency of photocatalytic decomposition of pollutants is greatly improved (Anieuddh et al., 2011; Liu et al., 2012; Pan et al., 2012). Up to date, SiC/C composites are mainly fabricated by a heat-treatment under high temperatures, a carbon ther-

This work was financially supported from National Natural Science Foundations of China (No. 51331006, 51271044 and 51171033).

(a) This is an open-access article distributed under the terms of the Creative Commons Attribution Non-Commercial License (http://creativecommons.org/licenses/by-nc/4.0) which permits unrestricted noncommercial use, distribution, and reproduction in any medium, provided the original work is properly cited.

Copyrights @ 2015 by Korean Society of Microscopy 
mal reduction or the vapor deposition method. Zhu et al. (2012) synthesized the graphene packaged $\mathrm{SiC}$ by sintering $\mathrm{SiC}$ under a low pressure and high temperature; George et al. (2010) prepared the microporous $\mathrm{SiC/C}$ composites by carbon thermal reduction of polysilsesquioxanes gel; Kim et al. (2003) reported that the aligned $\mathrm{SiC} / \mathrm{C}$ coaxial nanocables were synthesized via the direct growth of $\mathrm{SiC}$ nanowires from silicon substrates and subsequent carbon deposition by pyrolysis of methane. Nevertheless, the preparation of $\mathrm{SiC} /$ $\mathrm{C}$ nanocomposites by the arc-discharge plasma and their photocatalytic performances have rarely been investigated.

In this work, a series of $\mathrm{SiC} / \mathrm{C}$ nanocomposite powders have been synthesized by the arc-discharge plasma (Dong et al., 1999; Lei et al., 2009). The structures, compositions and morphologies of these nanocomposites were analyzed by means of X-ray diffraction (XRD) and scanning electron microscope (SEM). The photocatalytic degradation of methylene blue (MB) by such $\mathrm{SiC} / \mathrm{C}$ nancomposite powders were also examined.

\section{MATERIALS AND METHODS}

The arc-discharge plasma method was used to synthesize SiC/ $\mathrm{C}$ nanocomposite powders. In any case, the micron-sized $\mathrm{Si}$ powders were used as the source of silcon. Firstly, to mix the micro-sized powders of graphite and silicon, then compress the mixture into a block which was then put into a graphite crucible to act as the anode of arc-discharge, while a graphite rod served as the cathode. Secondly, after the chamber was evacuated, a mixture of hydrogen and argon was introduced as the source of hydrogen plasma and the condensation gas, respectively. Finally, to ignite the arc and maintain the current at 90 A. Priority to take the nanopowders out from the chamber, a passivation process was necessary on the nanoparticles for stabilization.

In this paper, three carbon sources, i.e., solid graphite, gaseous methane $\left(\mathrm{CH}_{4}\right)$ or liquid ethanol, were employed to synthesize the $\mathrm{SiC} / \mathrm{C}$ nanocomposite powders. Table 1 shows the preparation conditions of carbon sources and evaporation atmospheres for $\mathrm{SiC} / \mathrm{C}$ nanopowders. In the case of solid carbon source, the molar ratios of graphite and silicon micropowders were fixed as 1.0:0.1, 1.0:0.9 and 1.0:1.0, accordingly the corresponding nanopowder samples are marked as S1, S2 and S3, respectively. As an auxiliary carbon source, the methane gas has been also applied as the atmosphere for evaporation of the solid mixture of graphite and silicon (the molar ratio of 1.0:0.9) and the produced nanopowders is labelled as SG1. The SiC/C nanopowder samples synthesized by using of unitary methan gas or liquid alcohol are labelled as G1 or L1, respectively. The nanopowderes were characterized by means of XRD, SEM and transmission electron microscopy (TEM). The photocatalytic performances of SiC/C nanopowders were also measured according to following steps: to weigh up $0.5 \mathrm{~g}$ of nanopowders and put them into the $\mathrm{MB}$ solution with concentration of $10 \mathrm{mg} / \mathrm{L}$, then sonicate for 5 minutes and stir for 15 minutes with a magnetic stirrer under black light condition; then place the solution under an ultraviolet lamp (power of $300 \mathrm{~W}$ ) and continue stirring. To sample $1.5 \mathrm{~mL}$ of the soltion in an interval of 20 minutes and centrifuge them for 10 minutes with speed of 8,000 r/s. The supernatant fluid was taken to test its absorbance by ultraviolet visible light. Using the calculated concentration, the degradation curve of MB was drawn.

\section{RESULTS AND DISCUSSION}

\section{Solid Carbon Source for SiC/C Nanopowders}

Fig. 1 shows XRD patterns of samples S1, S2 and S3, all of these $\mathrm{SiC} / \mathrm{C}$ nanopowders were prepared by using of solid graphite source, however different in molar ratios of $\mathrm{C}$ and $\mathrm{Si}$ in the raw materials. As it can be anticipated, the sample S1 (Fig. 1 'a') has a main phase of graphite due to the abundant content of carbon in its raw material, and secondary phase of $\beta$-SiC with cubic lattice (JCPDS No. 291129). The sample S2 (Fig. 1 'b') consists of almost single phase of $\beta$-SiC, nevertheless a small quantity of free graphite and silicon phases exist in sample S2 (Fig. 1 ' $c$ '). Thus, the optimum composition range could be determined for $\mathrm{C}$ and Si elements, that is the content of carbon should be a little higher than the stoichiometry of $\mathrm{SiC}$ and then near single

Table 1. The raw sources and evaporation atmospheres for $\mathrm{SiC} / \mathrm{C}$ nanocomposite powders

\begin{tabular}{|c|c|c|c|c|c|c|}
\hline \multirow{2}{*}{$\begin{array}{c}\text { Silicon source } \\
\text { Si micropowders }\end{array}$} & \multicolumn{3}{|c|}{ Carbon sources } & \multicolumn{2}{|c|}{ Atmospheres (MPa) } & \multirow{2}{*}{ Samples markec } \\
\hline & Graphite micropowders & $\mathrm{CH}_{4}(\mathrm{MPa})$ & Alcohol & $\mathrm{H}_{2}$ & $\mathrm{Ar}$ & \\
\hline \multirow[t]{4}{*}{ Si micropowders } & $\mathrm{C} / \mathrm{Si}=1.0 / 0.1$ (molar) & - & - & & & S1 \\
\hline & $\mathrm{C} / \mathrm{Si}=1.0 / 0.9$ (molar) & - & - & 0.025 & 0.05 & S2 \\
\hline & $\mathrm{C} / \mathrm{Si}=1.0 / 1.1$ (molar) & - & - & & & S3 \\
\hline & $\mathrm{C} / \mathrm{Si}=1.0 / 0.9$ (molar) & 0.01 & - & 0.01 & 0.04 & SG1 \\
\hline Si micropowders & - & 0.02 & - & 0.01 & - & G1 \\
\hline Si micropowders & - & - & $\sqrt{ }$ & 0.01 & 0.04 & L1 \\
\hline
\end{tabular}


phase of $\mathrm{SiC}$ or carbon-riched $\mathrm{SiC} / \mathrm{C}$ nanopowders can be obtained. From the width of diffraction peaks of $\mathrm{SiC}$, its grain size can be estimated. For example, the mean size of crystal $\mathrm{SiC}$ in sample $\mathrm{S} 2$ is $\sim 50 \mathrm{~nm}$, which is calculated by Scherrer formula $\mathrm{D}_{h k l}=\mathrm{K} \lambda /(\beta \cdot \cos \theta)$ and would be much more bigger than that in sample S1.

Fig. 2 display SEM and TEM images of samples S1, and S2 and S3, respectively. It is found that morphologies of SiC/ $\mathrm{C}$ nanopowders are quite different each others due to their diverse compositions in raw materials. As shown in Fig. 2A, one-dimensional carbon nanostructures (Cai et al., 2012) are observed in S1 which has a rich-carbon content, that means a great deal of carbon can result in an anisotropic growth of nanorods in existence of $\mathrm{SiC}$ nanostructures; Sample $\mathrm{S} 2$ is nearly single phase of $\mathrm{SiC}$, nonetheless exhibits the complicated nanostructures of nanoparticles and claddings, as shwon in Fig. 2B. The possible formation mechanism is as follows: the solid sources of silicon and graphite are co-

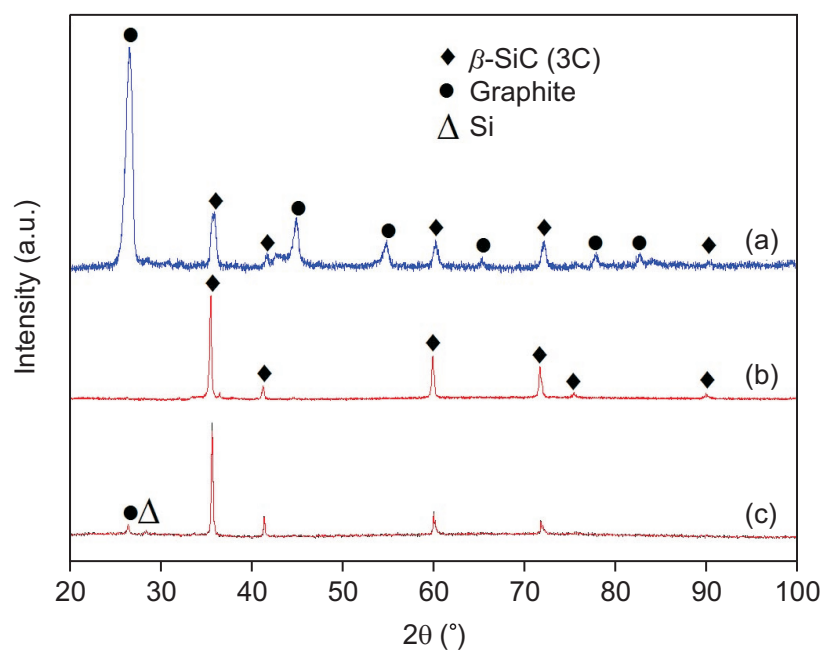

Fig. 1. X-ray diffraction patterns of $\mathrm{SiC} / \mathrm{C}$ nanocomposite powders using solid graphite and silicon sources: (a) sample $\mathrm{S1}, \mathrm{C}: \mathrm{Si}=1.0: 0.1$ in molar ratio; (b) sample $\mathrm{S} 2, \mathrm{C}: \mathrm{Si}=1.0: 0.9$ in molar ratio; and (c) sample $\mathrm{S3}$, $\mathrm{C}: \mathrm{Si}=1.0: 1.0$ in molar ratio. evaporated by arc-discharge to be the high-energy atomic states, and react to form $\mathrm{SiC}$. The higher atomic concentration and temperature gradient around the region of arc plasma promote the migration of $\mathrm{C}$ and $\mathrm{Si}$ atoms or $\mathrm{SiC}$ nucleus, subsequently grow into nanostructures with diverse morphologies and shapes. As the amount of graphite in excess of the stoichiometry of $\mathrm{SiC}$, the carbon-coated or -mixed $\mathrm{SiC} /$ $\mathrm{C}$ nanocomposite could be fabricated. The nanopowders tend to aggregate together owing to its higher surface energy with larger specific surface area, consequently, the $\mathrm{SiC} / \mathrm{C}$ nanoomposites with various shapes emerge. In sample S3, the little amount of free Si and graphite confirmed by XRD pattern (Fig. 1 ' $c$ ') can be recognised as the rods or small round-shaped particles as shown in Fig. 2C. The dominant phase of $\mathrm{SiC}$ in sample $\mathrm{S} 3$ has nanostructures with the irregular shapes or facets.

For a photocatalytic material, the intrinsic characteristic, structure and morphology have great effects on its photocatalytic performance (Georg \& Josef, 2005; Yu et al., 2008; Zhu et al., 2012). In comparison to the nanomaterials with a uniform distribution of particle's size, the SiC/C nanocomposites with a wider distribution in its particle's size may have a broader light absorbance and a better photocatalytic performance (Saber et al., 2010). In this work, sample S2 is chosen to test its photocatalytic property which will be presented in section 3.4 together with the results from other kinds of $\mathrm{SiC} / \mathrm{C}$ nanopowders synthesized by gaseous methane or liquid ethanol.

\section{Gaseous Carbon Source for SiC/C Nanopowders}

Gaseous carbon source of methane, meanwhile pure solid $\mathrm{Si}$ source, were used to produce the $\mathrm{SiC} / \mathrm{C}$ nanopowders (sample G1). For comparison, using the same gaseous $\mathrm{CH}_{4}$ as an atmosphere, however a mixture of graphite and silicon micropowders was used as the solid source to form the SiC/ C nanopowders (sample GS1). It means that two kinds of carbon sources (gas and solid) have been adopted here. Fig. 3 show the XRD patterns of samples G1 and SG1, respectively.
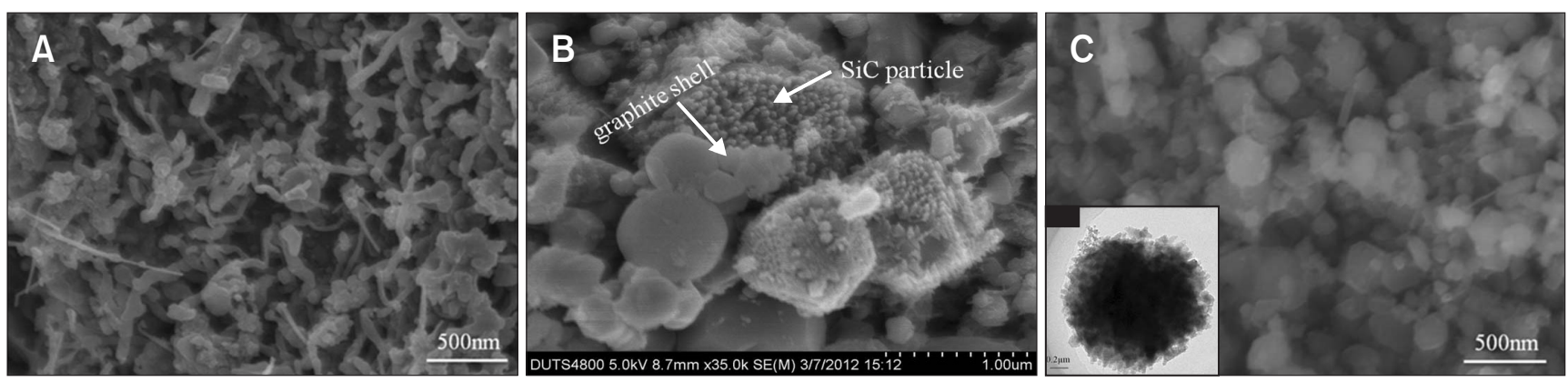

Fig. 2. Scanning electron microscope (A-C) and transmission electron microscopy (inset) images of SiC/C nanocomposite powders. (A) Sample S1. (B) Sample S2. (C, inset) Sample S3. 
As shown in Fig. 3 'a', main phase of $\mathrm{SiC}$ has created as using of the gaseous carbon source, except for a small amount of silicon and graphite. The sharp and strong diffraction peaks of SiC imply a well-crystallized nanostructure with a bigger grain size. Such SiC/C nanopowders (G1) is similar to sample S3 (using the unitary solid carbon source) in the characters. As shown in Fig. 4A for SEM images of sample G1, it is an assembly of nanoparticles due to their high surface energies. It can be concluded that both of carbon sources regardless in solid or gaseous state are equivalent in formation of SiC/C

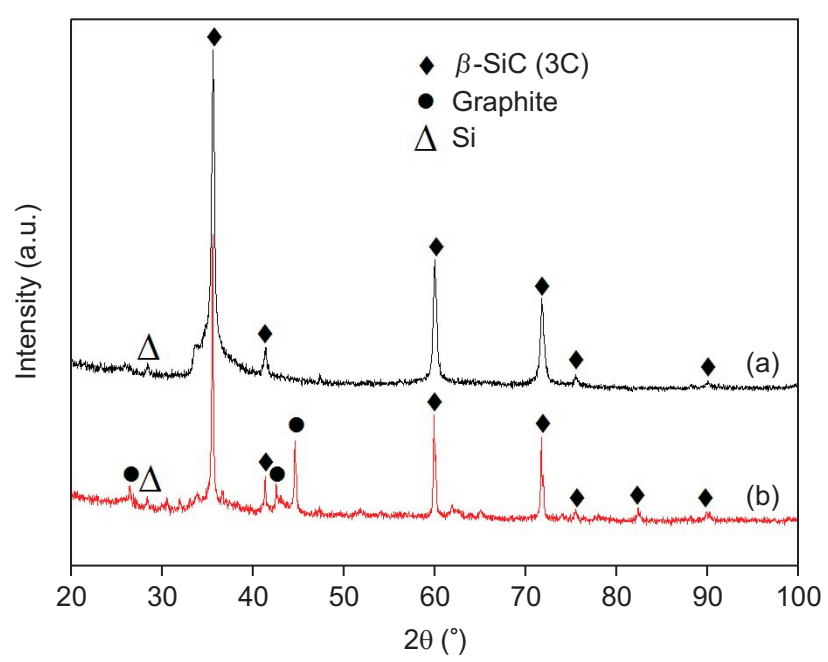

Fig. 3. X-ray diffraction patterns of $\mathrm{SiC} / \mathrm{C}$ nanocomposite powders: (a) sample G1 and (b) sample SG1. nanopowders, but the composition and morphology of SiC/C nanopowder could be different, depending on the manner to control or adjust.

For the case of using an extra solid carbon together with $\mathrm{CH}_{4}$ (gaseous carbon), the resultant $\mathrm{SiC} / \mathrm{C}$ nanopowders (GS1) is shown in Fig. 3 ' b'. it is found an excess graphite exists in this product of GS1. It is worth to note that the diffraction peak of graphite at $\sim 45^{\circ}$ becomes strong, implying an anisotropic growth of graphite which is further proved by the nanosized flakes shown in SEM and TEM images (Fig. 4C). The flakes

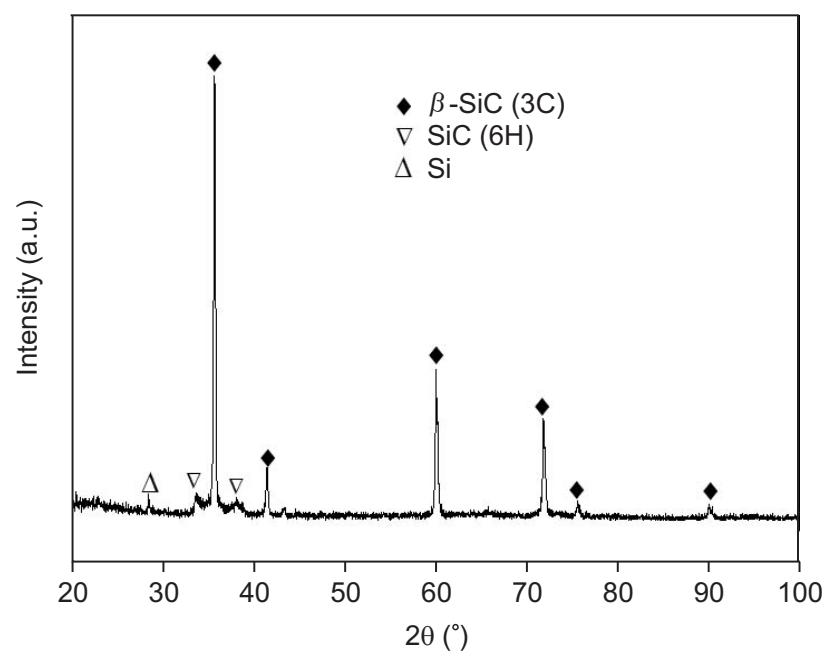

Fig. 5. X-ray diffraction pattern of SiC/C nanocomposite powders sample L1.
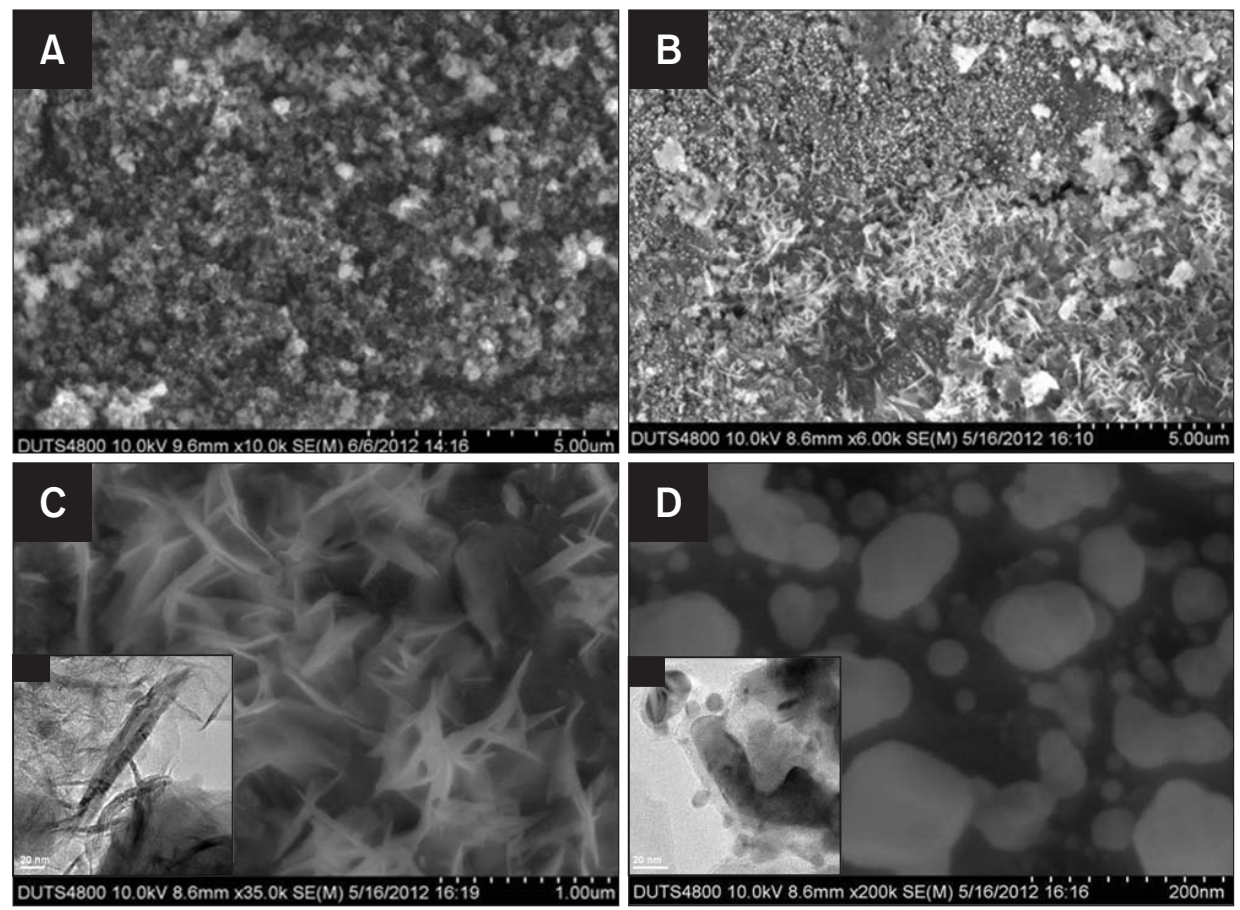

Fig. 4. Scanning electron microscope (AD) and transmission electron microscopy (insets in Fig. 4C and D) images of $\mathrm{SiC} /$ C nanocomposite powders. (A) Sample G1. (B-D, insets in Fig. 4C and D) Sample SGl. 
are actually of graphite nanosheets or graphenes, and had been successfully fabricated by arc-discharge method (Guo et al., 2013). Fig. 4B-D and insets in Fig. 4C and D show SEM and TEM images of these SiC/C nanopowders (GS1) by different magnifications. Besides the nano-flakes of graphite, a great amount of nanoparticles, such as $\mathrm{SiC}, \mathrm{Si}$ or graphite are also found.

\section{Liquid Carbon Source for SiC/C Nanopowders}

As presented in above sections, both of solid and gaseous carbon sources can be available to synthesize SiC/C nanopowders. Liquid ethanol has also been tested for the possibility to be a carbon source, and the product sample is marked as L1. As shown in Fig. 5, the XRD profile of sample L1 indicate that a main phase of $\beta$-SiC and a minor phase of $6 \mathrm{H}-\mathrm{SiC}$ (JCPDS No. 01-070-2550) which has hexagonal lattice and space group of $\mathrm{P} 63 \mathrm{mc}$. The morphologies of sample L1 are
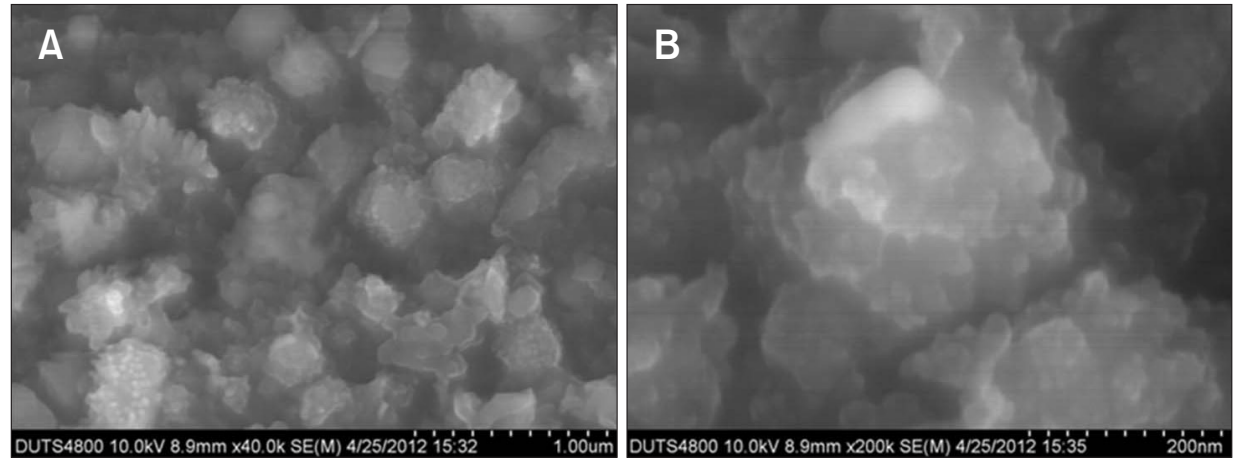

Fig. 6. (A, B) Scanning electron microscope images of $\mathrm{SiC} / \mathrm{C}$ nanocomposite powders sample L1.
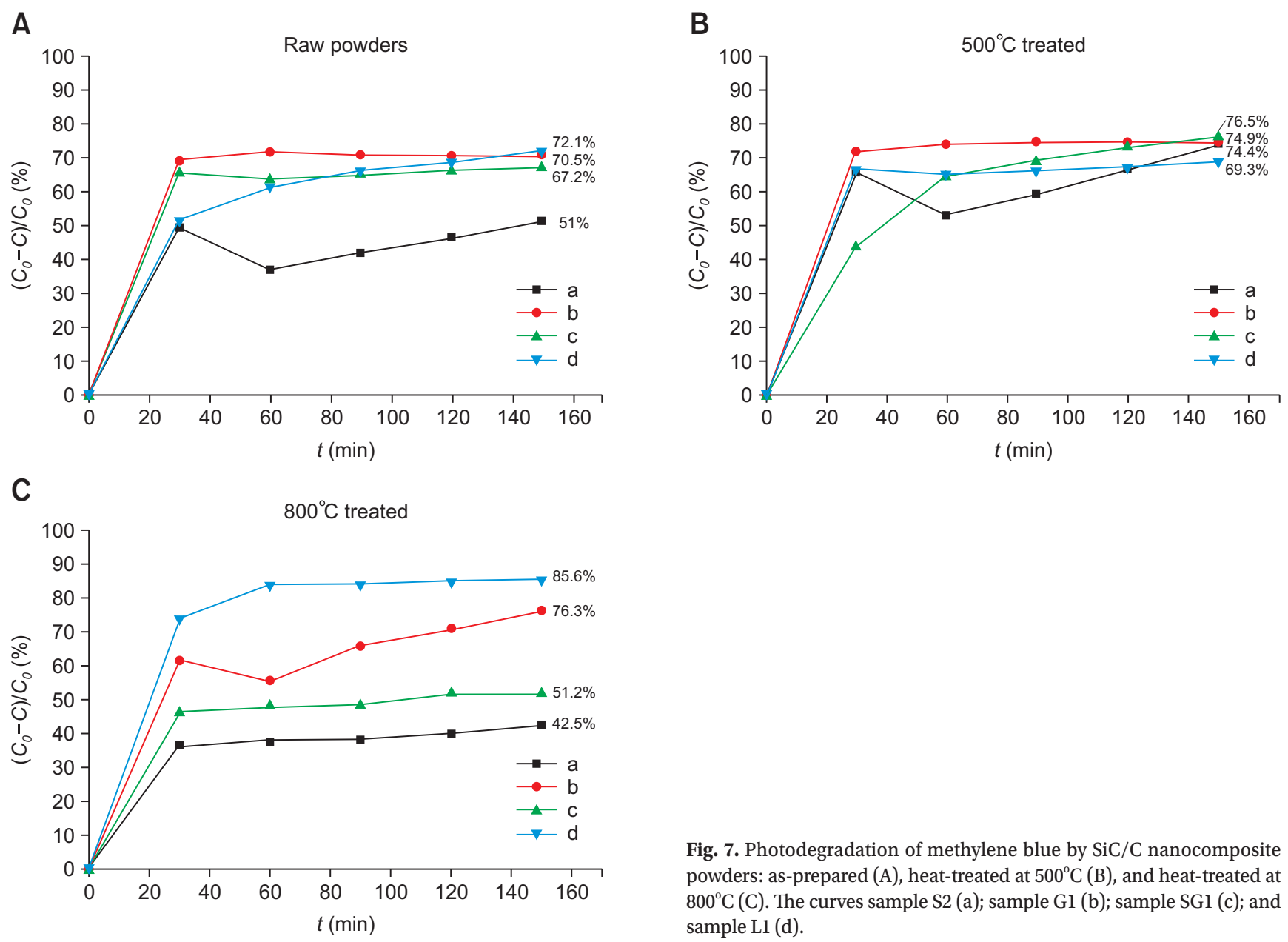

Fig. 7. Photodegradation of methylene blue by $\mathrm{SiC} / \mathrm{C}$ nanocomposite powders: as-prepared (A), heat-treated at $500^{\circ} \mathrm{C}(\mathrm{B})$, and heat-treated at $800^{\circ} \mathrm{C}(\mathrm{C})$. The curves sample S2 (a); sample G1 (b); sample SG1 (c); and sample Ll (d). 
tightly coalescent assemblies of fine particles with a petal-like shape in several tens of nanometers of its diameter (Fig. 6), it is quiet defferent from those in samples S1 3 and G1 (Fig. 2 and 4).

\section{Photocatalytic Performances of SiC/C Nanopowders}

The graphite layers or particles mixed in $\mathrm{SiC} / \mathrm{C}$ nanocomposite nanopowders can be altered or removed by a heattreatment in air. Thus the photocatalytic performances of $\mathrm{SiC} / \mathrm{C}$ nanocomposites can be controlled and improved. Four kinds of $\mathrm{SiC} / \mathrm{C}$ nanocomposite nanopowders, i.e., asprepared samples S2, G1, SG1 and L1, and their heat-treated ones were chosen to measure the photodegradation abilities on MB. As shown in Fig. 7, all the samples have the abilities to photodegrade $\mathrm{MB}$, moreover among them the sample G1 (by methane source) exhibits the beast performance in its as-prepared and $500^{\circ} \mathrm{C}$-treated ones; the sample L1 (by liquid alcohol source) is best in its $800^{\circ} \mathrm{C}$-treated one. The photocatalytic performances of samples S2 (by solid graphite source) and SG1 (by integration of solid and gaseous source), on the contrary, behave a significantly decrease after heattreatement at $800^{\circ} \mathrm{C}$.

The highest photodegradation rates for $\mathrm{MB}$ reach $74.9 \%$ (S1: $500^{\circ} \mathrm{C}$-treated), $76.3 \%$ (G1: $800^{\circ} \mathrm{C}$-treated), $76.5 \%$ (SG1: $500^{\circ} \mathrm{C}$-treated) and $85.6 \%\left(\mathrm{~L} 1: 500^{\circ} \mathrm{C}\right.$-treated), respectively. It is indicated that a themal-treatment in air is significant in the structural modification on $\mathrm{SiC} / \mathrm{C}$ nanocomposites and the improved photocatalytic performances. The reasons may be from two viewpoints: Firstly, on the one hand, the thickness of graphite in $\mathrm{SiC} / \mathrm{C}$ nanopowders become thinner by the heat-treatment, so more light incident on the junction regions favor to creat photoelectrons and holes. On the other hand, the time for photochemical reactions between the photogenerated carriers and pollutant molecules become shorter and greatly reduce the recombination probability for the electrons and holes. Secondly, by the heat-treatment, a certain quantity of $\mathrm{SiO}_{2}$ would appeare on the surface of $\mathrm{SiC}$. The combined oxygen in $\mathrm{SiO}_{2}$ may react with $\mathrm{H}_{2} \mathrm{O}$ to form a strong hydroxy group $\cdot \mathrm{OH}$ which can promote the photocatalytic decomposition of MB (Kang et al., 2007).

\section{CONCLUSIONS}

Three carbon sources, i.e., solid graphite, gaseous $\mathrm{CH}_{4}$ and liquid ethanol, have been successfully employed to synthesize $\mathrm{SiC} / \mathrm{C}$ nanocomposite powders by the arc-discharge plasma. In the case of solid sources, the optimum composition of raw material is that the content of carbon should be a little higher than the stoichiometry of $\mathrm{SiC}$ and then near single phase of $\mathrm{SiC}$ or carbon-riched $\mathrm{SiC} / \mathrm{C}$ nanopowders can be obtained. The gaseous carbon source is equivalent to the solid one in formation of SiC/C nanopowders, and favor to control the composition and morphology of SiC/C nanopowders. Liquid ethanol is also an alternative carbon source to synthesize $\mathrm{SiC} /$ $\mathrm{C}$ nanocomposite powders. It is consisted of a dominant cubic $\beta$-SiC and a minor hexagonal $6 \mathrm{H}-\mathrm{SiC}$ phase, and the morphology is tightly coalescent assembly of fine particles in petal-like shape with several tens of nanometers in diameter. Heat-treatment on the as-prepared $\mathrm{SiC} / \mathrm{C}$ nanocomposite powders in air is significant in the structural modification and the improved photocatalytic performances of $\mathrm{SiC} / \mathrm{C}$ nanopowders. The highest photodegradation rates for $\mathrm{MB}$ reach $74.9 \%$ (S1: $500^{\circ} \mathrm{C}$-treated), $76.3 \%$ (G1: $800^{\circ} \mathrm{C}$-treated), 76.5\% (SG1: $500^{\circ} \mathrm{C}$-treated) and $85.6 \%\left(\mathrm{~L} 1: 500^{\circ} \mathrm{C}\right.$-treated), for various kinds of $\mathrm{SiC} / \mathrm{C}$ nanopowders.

\section{CONFLICT OF INTEREST}

No potential conflict of interest relevant to this article was reported.

\section{REFERENCES}

Anieuddh M, Brian S, Liu G Q, and Wang L Z (2011) Nitrogen doped Sr2Ta2O7 coupled with graphene sheets as photocatalysts for increased photocatalytic hydrogen production. Nano 5, 3483-3492.

Cai X K, Cong H T, and Liu C (2012) Synthesis of vertically-aligned carbon nanotubes without a catalyst by hydrogen arc discharge. Carbon $\mathbf{5 0}$, 2726-2730.

Dong X L, Zhang Z D, Zhao X G, and Chuang Y C (1999) The preparation and characterization of ultrafine Fe -Ni particles. J. Mater. Res. 14, 398-406.

Fujishima A and Honga K (1972) Electrochemical photolysis of water at a semiconductor electrode. Nature 238, 37-38.

Georg W and Josef K (2005) Photocurrents and degradation rates on particulate TiO2 layers effect of layer thickness, concentration of oxidizable substance and illumination direction. Electrochimica Acta
50, 4498-4504

George H, Kazuyoshi K, Kazuki N, and Teiichi H (2010) A new route to monolithic macroporous $\mathrm{SiC} / \mathrm{C}$ composites from biphenylene-bridged polysilsesquioxane gels. Chem. Mater. 22, 2541-2547.

Guo G F, Huang H, Xue F H, Liu C J, Yu H T, Quan X, and Dong X L (2013) Electrochemical hydrogen storage of the graphene sheets prepared by DC arc-discharge method. Surf. Coat. Technol. 228, 120-125.

Isaias J R, Edgar M, Leticia M, Torres M, and Christian G S (2013) Short time deposition of $\mathrm{TiO} 2$ nanoparticles on $\mathrm{SiC}$ as photocatalysts for the degradation of organic dyes. Res. Chem. Inter. 39, 1523-1531.

Kang M G, Han H E, and Kim K J (1999) Enhanced photodecomposition of 4-chlorophenol in aqueous solution by deposition of CdS on TiO2. J. Photochem. Photobiol. A 125, 119-125. 
Kang Z H, Cha T A, Wong N B, and Zhang Z D (2007) Silicon quantum dots: a general photocatalyst for reduction, decomposition, and selective oxidation reactions. Am. Chem. Soc. 129, 12090-12091.

Kang Z, Liu Y, Tsang C, Fan X, and Wong N B (2009) Water-soluble silicon quantum dots with wavelength-tunable photoluminescence. Adv. Mater. 21, 661-664.

Kim H Y, Bae S Y, Kim N S, and Park J (2003) Fabrication of SiC-C coaxial nanocables: thickness control of $\mathrm{C}$ outer layers. Chem. Comm. $\mathbf{2 0}$, 2634-2635.

Lei J P, Huang H, Dong X L, Sun J P, and Lua B (2009) Formation and hydrogen storage properties of in situ prepared Mg-Cu alloy nanoparticles by arc discharge. Int. J. Hydrogen Energy 34, 8127-8134.

Liu H L, She G W, Mu L X, and Shi W S (2012) Porous SiC nanowire arrays as stable photocatalyst for water splitting under UV irradiation. Mater. Res. Bull. 47, 917-920.

Liu Y S, Ji G B, and Wang J Y (2012) Fabrication and photocatalytic properties of silicon nanowires by metal-assisted chemical etching: effect of $\mathrm{H} 2 \mathrm{O} 2$ concentration. Nanoscale Res. Lett. 7, 663.

Nacera M, Yannick C, Sabine S, Toufik H, Omar E, and Rabah B (2011) Photocatalytic activity of silicon nanowires under UV and visible light irradiation. Chem. Commun. 47, 991-993.

Nariki Y, Inoue Y, and Tanaka K (1990) Production of ultra fine SiC powder from SiC bulk by arc-plasma irradiation under different atmospheres and its application to photocatalysts. J. Mater. Sci. 25, 3101 3104.
Nicolas K, Valerie R, Francois G, and Marc J L (2004) A new TiO2- $\beta$-SiC material for use as photocatalyst. Mat. Lett. 58, 970-974.

Noboru O, Tatsuo F, Masakazu K, Takashi A, and Hirokatsu Y (2002) Growth of large high-quality SiC single crystals. J. Crystal Growth 237, 1180-1186.

Pan X, Zhao Y, Liu S,Carol L K, Wang S, and Fan Z Y (2012) Comparing graphene-TiO2 nanowire and graphene-TiO2 nanoparticle composite photocatalysts. Mater. Interf. 4, 3944-3950.

Saber A, Rasul M G, Wayde N M, Bromn R, and Hashib M A (2010) Heterogeneous photocatalytic degradation of phenols in wastewater: a review on current status and developments. Desalination 261, 3-18.

Shao M, Cheng L, and Zhang X (2009) Excellent photocatalysis of HFtreated silicon nanowires. J. Am. Chem. Soc. 131, 17738-17739.

Yu H T, Quan X, Chen S, Zhao H M, and Zhang Y B (2008) TiO2-carbon nanotube heterojunction arrays with a controllable thickness of TiO2 layer and their first application in photocatalysis. J. Photochem. Photobiol. A: Chem. 200, 301-306.

Zhou W M, Yan L J, and Wang Y (2006) SiC nanowires: a photocatalytic nanomaterial. Appl. Phy. Lett. 89, 13105-1-3.

Zhu K X, Guo L W, and Lin J J (2012) Graphene covered SiC powder as advanced photocatalytic material. Phy. Lett. 100, 023113-1 023113-4.

Zhu K X L, Guo W, and Lin J J (2012) Graphene covered SiC powder as advanced photocatalytic material. Phy. Lett. 100, 023113(1-4). 